\title{
Guaranteed Cost Control for Multirate Networked Control Systems with Both Time-Delay and Packet-Dropout
}

\author{
Qixin Zhu, ${ }^{1,2}$ Binbin Xie, ${ }^{2}$ and Yonghong $\mathrm{Zhu}^{3}$ \\ ${ }^{1}$ School of Mechanical Engineering, Suzhou University of Science and Technology, Suzhou 215009, China \\ ${ }^{2}$ School of Electrical and Electronic Engineering, East China Jiaotong University, Nanchang 330013, China \\ ${ }^{3}$ School of Mechanical and Electronic Engineering, Jingdezhen Ceramic Institute, Jingdezhen 333001, China
}

Correspondence should be addressed to Qixin Zhu; bob21cn@163.com

Received 26 November 2013; Revised 8 February 2014; Accepted 9 February 2014; Published 17 April 2014

Academic Editor: Xudong Zhao

Copyright (C) 2014 Qixin Zhu et al. This is an open access article distributed under the Creative Commons Attribution License, which permits unrestricted use, distribution, and reproduction in any medium, provided the original work is properly cited.

\begin{abstract}
Compared with traditional networked control systems, the sampling rates of the nodes are not the same in the multirate networked control systems (NCSs). This paper presents a new stabilization method for multirate NCSs. A multirate NCSs with simultaneous considering time-delay and packet-dropout is modeled as a time-varying sampling system with time-delay. The proposed Lyapunov function deceases at each input signal updating point, which is largely ignored in prior works. Sufficient condition for the stochastic mean-square stability of the multirate NCSs is given, and the cost function value is less than a bound. Numerical examples are presented to illustrate the effectiveness of the proposed control scheme.
\end{abstract}

\section{Introduction}

Feedback control systems where in the control loops are closed through real-time network are called Networked Control Systems (NCSs) [1]. Compared with the traditional control architecture, NCSs have many advantages such as high reliability, simple installation, and lower cost. Although the NCSs have many advantages, the applying of NCSs makes the system more complicated to analyze. Since the data is transmitted via network, there are two major problems of NCSs. Firstly, the network-induced delay occurs while transferring data between devices and shared medium. Secondly, unreliable network transmission may lead to packet dropout.

For these reasons, it is vital to study NCSs with networkinduced delay and packet dropout. Up to now, many good achievements have been investigated to deal with these problems. For the issue of time delay, the stability of NCSs with short random time delay was studied in [1]. The achievement in [1] was expanded for the situation of long time delay in [2]. The system was modeled into switch systems to investigate the stability of networked control systems in [3]. Packet dropout not only exists in time delay progress but also in transmission loss. For the problem of packet dropout, the stabilizing of controller was investigated in [4-18]. The models of NCSs in prior papers were divided into three cases: switch linear systems [6], asynchronous dynamical systems [9], and jump linear systems [13]. It is more complex to deal with the modeling and analysis for NCSs with both delay and packet dropout, compared with separately considering each other. In [15], the method of switched linear systems was applied in modeling for the NCSs of both packet dropout and network-induce delay in NCSs. Sufficient conditions for stochastic stability were discussed in [16]; what is more, packet dropout on both sides of sensor-to-controller and controller-to-sensor was modeled as two Markov chains.

It is important to ensure that the system possesses a strong robust performance. The guaranteed cost control is a good way to deal this problem, which guarantees the system performance affected uncertainty bellow the given performance index bound. The guaranteed cost control was first mooted in [19]. These years it has been applied to networked control system with time delay, and many issues have been developed for this item in [20-25].

Unfortunately, most aforementioned conclusions are under the following assumption: the sampling rates of each node in NCSs are the same. This brings convenience for the 


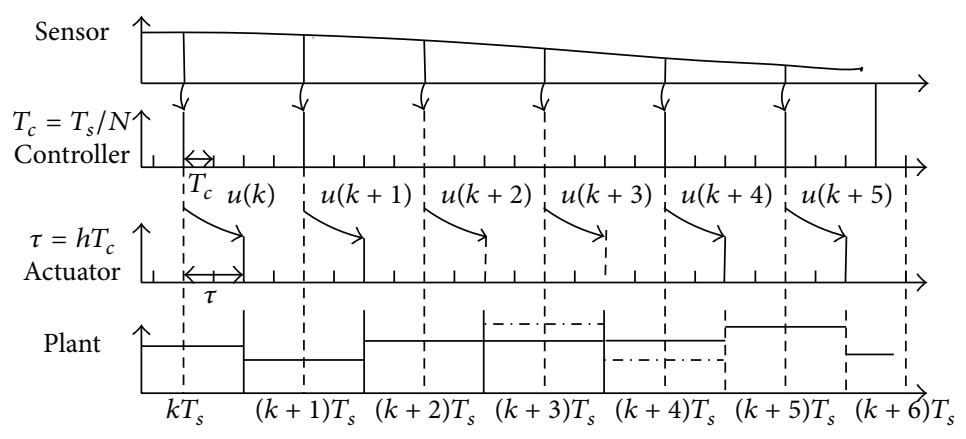

FIGURE 1: The timing diagrams of multirate NCSs.

theoretical research of NCSs; however, the sampling rate of each node is not identical in practical application. For the multirate network, the rates are not the same, the sampling period of sensor is $T_{s}$, and sampling period of the controller is $T_{c}, T_{s} \neq T_{c}$. In recent years, the investigations of multirate control system have made a great progress [26-36]. The NCSs are modeled as switched systems by using multirate method, when sensor, controller, and actuator are all event-driven, in [26], and the stability was analyzed. The exponential stability of multirate NCSs was analyzed including three cases of perfect transmission, delayed transmission, and time-varying transmission, in [28]. In [32], the condition of stabilizing controller of multirate NCSs was discussed by the way of using a V-K iteration algorithm. Controllability and observability of networked control systems with short time delay are analyzed in $[35,36]$.

It is nice to see that the network control systems theory has been widely applied to practical area. Two online schemes based on the data-driven fault-tolerant control (FTC) systems on the benchmark Tennessee Eastman process are presented in [37]. A subspace-aided data-driven approach for batch processes is proposed in [38]. A comparison between the basic data-driven methods for process monitoring and fault diagnosis (PM-FD) is provided in [39].

Published literature shows that many questions about guaranteed cost control for multirate NCSs with both time delay and packet dropout should be investigated. The main contributions of this paper are as follows. (1) A multirate NCSs with simultaneous consideration time delay and packet dropout are modeled as a time-varying sampling system with time delay. (2) The Lyapunov function deceases at each input signal updating point, which is largely ignored in prior works. Compared with traditional NCSs methods, it can yield less conservative. (3) State feedback controller of multirate NCSs, which render the multirate networked control systems stochastic mean square stable, is proposed. And the cost function value is less than a bound.

This paper is organized into four sections including the Introduction. Problem formulations and main assumptions were presented in Section 2. In Section 3, the guaranteed cost control of NCSs was discussed. The controller is proved to render the system stochastic mean square stable. An illustrative example is provided in Section 4.
Notations. The superscript " $T$ " stands for the transpose of a matrix. $R^{n}$ and $R^{n \times m}$ denote the $n$ dimensional Euclidean space and the set of all $n \times m$ real matrices, respectively. $\|\cdot\|$ stands for the Euclidean norm. $I$ and 0 stand for identified matrices and zero matrices with appropriate dimensions, respectively. The notation $X>0(X \geq 0)$ means that the matrix $X$ is positive definite ( $X$ is semipositive definite). $I$ is the identity matrix of appropriate dimensions. $\left[\begin{array}{ll}X & Z \\ * & Y\end{array}\right]$ denotes a symmetric matrix, where $*$ denotes the entries implied by symmetry.

\section{Problem Formulation}

It is assumed that the controlled process is a linear timeinvariant system, which can be expressed as

$$
\begin{gathered}
\dot{x}(t)=A^{c} x(t)+B^{c} u(t)+E^{c} v(t), \\
z(t)=C x(t)+H v(t),
\end{gathered}
$$

where $x(t) \in R^{n}, u(t) \in R^{m}, v(t) \in R^{q}$, and $A^{c}, B^{c}, E^{c}, C, H$ are matrices of appropriate sizes and $v(t)$ is white noise with zero mean. The sampling period of the sensor is noted as $T_{s}$, and the sampling periods of controller and actuator are the same, noted as $T_{c} . T_{c}=T_{s} / N$ and $N$ are a positive integer not less than 2. That is to say, the controller and actuator have a higher sampling frequency than the sensor. In order to facilitate the discussion, the delay of the system is considered to be a constant short delay, in this paper. The information transmission sequence of multirate NCSs is in Figure 1.

In convenience of investigation, we make the following rational assumptions.

(A1) The sensor, the controller, and the actuator are all time-driven, sensor-to-controller delay is denoted by $\tau_{s c}$, and the delay of controller-to-actuator is denoted by $\tau_{c a}$. The time delay in the system is $\tau=\tau_{s c}+\tau_{c a}=$ $h T_{c}, h \leq N$, and $h$ is a positive integer.

(A2) The number of successive packet dropouts is upper bounded, and the bound is denoted a known constant d.

(A3) The system adopts the Zero Order Hold ( $\mathrm{ZOH})$ strategy. 
In the multirate networked control systems, the inputs of sampling interval are different from sampling interval to interval. The model of multirate NCSs is described as follows.

Case $S_{i}^{(0)}$. There is no packet dropout which occurs in the current sampling interval. Such as in the interval $[(k+$ 5) $\left.T_{s},(k+6) T_{s}\right]$

$$
\begin{aligned}
x\left[(k+1) T_{s}\right]= & A x\left(k T_{s}\right) \\
& +\left(\int_{k T_{s}}^{k T_{s}+h T_{c}} e^{A^{c}\left[(k+1) T_{s}-\eta\right]} B^{c} d \eta\right) \\
& \times u\left[(k-1) T_{s}\right] \\
& +\left(\int_{k T_{s}+h T_{c}}^{(k+1) T_{s}} e^{A^{c}\left[(k+1) T_{s}-\eta\right]} B^{c} d \eta\right) u\left(k T_{s}\right) \\
& +E^{c} v\left(k T_{s}\right) \\
= & A x\left(k T_{s}\right)+\left(\int_{0}^{(N-h) T_{c}} e^{A^{c} \eta_{1}} B^{c} d \eta_{1}\right) u\left(k T_{s}\right) \\
& +\left(\int_{(N-h) T_{c}}^{N T_{c}} e^{A^{c} \eta_{1}} B^{c} d \eta_{1}\right) u\left[(k-1) T_{s}\right] \\
& +E v\left(k T_{s}\right) \\
= & A x\left(k T_{s}\right)+\left(B_{1}+B_{2}+\cdots+B_{N-h}\right) u\left(k T_{s}\right) \\
& +\left(B_{N-h+1}+B_{N-h+2}+\cdots+B_{N}\right) \\
& \times u\left[(k-1) T_{s}\right]+E v\left(k T_{s}\right),
\end{aligned}
$$

where

$$
\begin{aligned}
& A=e^{A^{c} T_{s}}, \quad B_{1}=\int_{0}^{T_{c}} e^{A^{c} \eta_{1}} B^{c} d \eta_{1}, \\
& B_{k}=D^{k-1} B_{1}, \quad D=\int_{0}^{T_{c}} e^{A^{c} \eta_{1}} d \eta_{1}, \quad 1 \leq k \leq N, \\
& E=\int_{0}^{T_{s}} e^{A^{c} \eta_{1}} E^{c} d \eta_{1} .
\end{aligned}
$$

Case $S_{i}^{(1)}$. There are $i$ successive packet dropouts in the current sampling interval. Such as in interval $\left[(k+2) T_{s},(k+3) T_{s}\right]$ to interval $\left[(k+3) T_{s},(k+4) T_{s}\right]$. The inputs of actuator in this period are the latest effective inputs,

$$
\begin{aligned}
x\left[(k+1) T_{s}\right]= & A x\left(k T_{s}\right) \\
& +\left(\int_{k T_{s}}^{k T_{s}+T_{s}} e^{A^{c}\left[(k+1) T_{s}-\eta\right]} B^{c} d \eta\right) \\
& \times u\left[(k-i) T_{s}\right]+E v\left(k T_{s}\right) \\
= & A x\left(k T_{s}\right)+\left(B_{1}+B_{2}+\cdots+B_{N}\right) \\
& \times u\left[(k-i) T_{s}\right]+E v\left(k T_{s}\right) .
\end{aligned}
$$

Case $S_{i}^{(2)}$. There is no packet dropout within the current sampling interval, but the last $i$ times sampling intervals packet dropouts. Such as in the interval $\left[(k+3) T_{s},(k+4) T_{s}\right]$

$$
\begin{aligned}
x\left[(k+1) T_{s}\right]= & A x\left(k T_{s}\right) \\
& +\left(\int_{0}^{(N-h) T_{c}} e^{A^{c} \eta_{1}} B^{c} d \eta_{1}\right) u\left(k T_{s}\right) \\
& +\left(\int_{(N-h) T_{c}}^{N T_{c}} e^{A^{c} \eta_{1}} B^{c} d \eta_{1}\right) u\left[(k-1) T_{s}\right] \\
& +E v\left(k T_{s}\right) \\
= & A x\left(k T_{s}\right)+\left(B_{1}+B_{2}+\cdots+B_{N-h}\right) u\left(k T_{s}\right) \\
& +\left(B_{N-h+1}+B_{N-h+2}+\cdots+B_{N}\right) \\
& \times u\left[(k-i-1) T_{s}\right]+E v\left(k T_{s}\right) .
\end{aligned}
$$

Definition 1. Let $\varsigma=\left(i_{1}, i_{2}, \ldots, i_{k}\right\}$ denote the point of each input signal arrived,

$$
\xi_{k}=\left\{i_{k+1}-i_{k}\right\} \in\{1,2,3, \ldots, d\}, \quad d=\max \left(i_{k+1}-i_{k}\right) .
$$

Definition 2. We assume the packet dropout progress on the basis of a discrete-time Markova chain process, the mode of transition probabilities:

$$
p_{\xi_{k}}=\operatorname{Pr}\left(i_{k+1}=i+\xi_{k} \mid i_{k}=i\right)>0, \quad i \in \varsigma,
$$

where $\sum_{\xi_{k}=1}^{d} p_{\xi_{k}}=1$.

The controlled process can be rewritten as

$$
\begin{aligned}
x\left(i_{k+1}\right)= & A^{\xi_{k}} x\left(i_{k}\right)+\Gamma_{0}\left(\tau_{k}\right) u\left(i_{k}\right) \\
& +\left[\Gamma_{1}\left(\tau_{k}\right)+\delta_{\xi_{k}}\left(\sum_{0}^{\xi_{k}} A^{i}\right) \Gamma_{2}\left(\tau_{k}\right)\right] u\left(i_{k}-\xi_{k-1}\right) \\
& +E v\left(i_{k}\right),
\end{aligned}
$$

where $\delta_{\xi_{k}}=1$ if $\xi_{k}>1$, otherwise $\delta_{\xi_{k}}=0 . A^{\xi_{k}}=$ $\underbrace{A \cdot A \cdot A}_{\xi_{k}}, \Gamma_{0}\left(\tau_{k}\right)=\sum_{i=1}^{N-h} B_{i}$,

$$
\Gamma_{1}\left(\tau_{k}\right)=\sum_{i=N-h+1}^{N} B_{i}, \quad \Gamma_{2}\left(\tau_{k}\right)=\sum_{i=1}^{N} B_{i} .
$$

The state-based control scheme for the system (7) is described by

$$
u(k)=K x(k),
$$


where $K \in R^{m \times n}$ is the controller gain. Substituting (9) into (7) results in the following system:

$$
\begin{aligned}
x\left(i_{k+1}\right)= & \left(A^{\xi_{k}}+\Gamma_{0} K\right) x\left(i_{k}\right) \\
& +\left(\Gamma_{1}\left(\tau_{k}\right)+\delta_{\xi_{k}}\left(\sum_{0}^{\xi_{k}} A^{i}\right) \Gamma_{2}\right) K x\left(i_{k}-\xi_{k-1}\right) \\
& +E v\left(i_{k}\right),
\end{aligned}
$$

where $\tau_{k}$ is omitted here.

\section{Main Results and Proofs}

Firstly, we introduce the following lemmas and definitions, which will be cited for the proofs in this section.

Definition 3. The closed-loop networked control systems (10) are stochastic mean-square stable if when $v\left(i_{k}\right)=0, x_{0}=x(0)$ such that

$$
E\left(\sum_{0}^{\infty}\left\|x\left(i_{k}\right)\right\|^{2}\right)<\infty
$$

Definition 4. Our object is to design a controller such that the closed loop networked system with both packet dropout and time delay is stochastic mean-square stable and satisfies $H_{\infty}$ performance constraint $\gamma$. That is to say (10) satisfies the following three conditions simultaneously.

(N1) The closed loop networked control systems (10) are stochastic mean-square stable.

(N2) For system (10), the defined cost function:

$$
J=E\left\{\sum_{k=0}^{\infty} x^{T}\left(i_{k}\right) S x\left(i_{k}\right)+u^{T}\left(i_{k}\right) R u\left(i_{k}\right)\right\}
$$

satisfies $J \leq J^{*}$. $J^{*}$ is a constant, where $S>0, R>0$.

(N3) Under the zero-initial condition, for all nonzero $v\left(i_{k}\right)$, the controlled output $z_{i_{k}}$ satisfies

$$
\sum_{k=0}^{\infty} E\left(z_{i_{k}}^{T} z_{i_{k}}\right)-\gamma^{2} v_{i_{k}}^{T} v_{i_{k}}<0
$$

Lemma 5 (Schur complement). For given a matrix $S=$ $\left[\begin{array}{ll}S_{11} & S_{12} \\ S_{12}^{T} & S_{22}\end{array}\right]$, where $S_{11}, S_{12}$ are square matrices, the following conditions are equivalent:

(1) $S<0$;

(2) $S_{11}<0, \quad S_{22}-S_{12}^{T} S_{11}^{-1} S_{12}<0$;

(3) $S_{22}<0, \quad S_{11}-S_{12} S_{22}^{-1} S_{12}^{T}<0$.
Theorem 6. For the system (10), if positive definite matrixes exist $P, Q>0$, such that

$$
\begin{aligned}
& \text { (1) } 2 G^{T}(P+Q) G-P+S+K^{T} R K<0, \\
& \text { (2) } 2 H_{\xi_{k}}^{T}(P+Q) H_{\xi_{k}}-Q<0,
\end{aligned}
$$

where $G=A^{\xi_{k}}+\Gamma_{0} K, H_{\xi_{k}}=\left[\Gamma_{1}+\delta_{\xi_{k}}\left(\sum_{i=0}^{\xi_{k}} A^{i}\right) \Gamma_{2}\right] K$. Then the system (10) with the controller (9) is stochastic meansquare stable and the cost function value is less than a bound. The corresponding cost function satisfies $J<x^{T}\left(i_{0}\right) P x\left(i_{0}\right)+$ $x^{T}\left(i_{-1}\right) Q x\left(i_{-1}\right)$.

Proof. Define a Lyapunov function as

$$
\begin{aligned}
& V_{1}\left(i_{k}\right)=x^{T}\left(i_{k}\right) P x\left(i_{k}\right), \\
& V_{2}\left(i_{k}\right)=\sum_{s=\xi_{k-1}}^{i_{k}-1} x^{T}\left(i_{k}-s\right) Q x\left(i_{k}-s\right), \\
& V\left(i_{k}\right)=\sum_{j=1}^{2} V_{j}\left(i_{k}\right),
\end{aligned}
$$

where $P>0, Q>0$

$$
\Delta V\left(i_{k}\right)=E\left[V\left(i_{k+1}\right)-V\left(i_{k}\right)\right]=\sum_{j=1}^{2} E\left(\Delta V_{j}\left(i_{k}\right)\right) .
$$

Along the solution of (9), $E\left(\Delta V_{1}\left(i_{k}\right)\right), E\left(\Delta V_{2}\left(i_{k}\right)\right)$ takes the form of

$$
\begin{aligned}
E\left(\Delta V_{1}\left(i_{k}\right)\right)= & \sum_{\xi_{k}=1}^{d} p_{\xi_{k}}\left[G x\left(i_{k}\right)+H_{\xi_{k i}} x\left(i_{k}-\xi_{k-1}\right)\right]^{T} P \\
& \times\left[G x\left(i_{k}\right)+H_{\xi_{k}} x\left(i_{k}-\xi_{k-1}\right)\right] \\
& -x^{T}\left(i_{k}\right) P x\left(i_{k}\right), \\
E\left(\Delta V_{2}\left(i_{k}\right)\right) \leq & \sum_{\xi_{k}=1}^{d} p_{\xi_{k}}\left[G x\left(i_{k}\right)+H_{\xi_{k}} x\left(i_{k}-\xi_{k-1}\right)\right]^{T} Q \\
& \times\left[G x\left(i_{k}\right)+H_{\xi_{k}} x\left(i_{k}-\xi_{k-1}\right)\right] \\
& -\sum_{\xi_{k}=1}^{d} p_{\xi_{k}} x^{T}\left(i_{k}\right) Q x\left(i_{k}\right) .
\end{aligned}
$$

By (17) we can obtain

$$
\begin{aligned}
\Delta V\left(i_{k}\right) \leq & \sum_{\xi_{k}=1}^{d} p_{\xi_{k}}\left[G x\left(i_{k}\right)+H_{\xi_{k}} x\left(i_{k}-\xi_{k-1}\right)\right]^{T}(P+Q) \\
& \times\left[G x\left(i_{k}\right)+H_{\xi_{k}} x\left(i_{k}-\xi_{k-1}\right)\right] \\
& -x^{T}\left(i_{k}\right) P x\left(i_{k}\right) \\
& -\sum_{\xi_{k}=1}^{d} p_{\xi_{k}} x^{T}\left(i_{k}-\xi_{k-1}\right) Q x\left(i_{k}-\xi_{k-1}\right) .
\end{aligned}
$$


It is easy to obtain

$$
\begin{aligned}
\sum_{\xi_{k}=1}^{d} p_{\xi_{k}}\left[G x\left(i_{k}\right)+H_{\xi_{k}} x\left(i_{k}-\xi_{k-1}\right)\right]^{T}(P+Q) & \\
& \times\left[G x\left(i_{k}\right)+H_{\xi_{k}} x\left(i_{k}-\xi_{k-1}\right)\right] \\
= & \sum_{\xi_{k}=1}^{d} p_{\xi_{k}}\left[x^{T}\left(i_{k}\right) G^{T}(P+Q) H_{\xi_{k}} x\left(i_{k}-\xi_{k-1}\right)\right] \\
& +\sum_{\xi_{k}=1}^{d} p_{\xi_{k}}\left[x^{T}\left(i_{k}-\xi_{k-1}\right) H_{\xi_{k}}^{T}(P+Q) G x\left(i_{k}\right)\right] \\
& +\sum_{\xi_{k}=1}^{d} p_{\xi_{k}}\left[x^{T}\left(i_{k}\right) G^{T}(P+Q) G x\left(i_{k}\right)\right] \\
& +\sum_{\xi_{k}=1}^{d} p_{\xi_{k}}\left[x^{T}\left(i_{k}-\xi_{i_{k-1}}\right) H_{\xi_{k}}^{T}(P+Q) H_{\xi_{k}} x\left(i_{k}-\xi_{i_{k-1}}\right)\right] .
\end{aligned}
$$

By Lemma 5, $\left[G x\left(i_{k}\right), H_{\xi_{k}} x\left(i_{k}-\xi_{k-1}\right)\right]^{T}\left[\begin{array}{cc}-P-Q & P+Q \\ P+Q & -P-Q\end{array}\right]\left[G x\left(i_{k}\right)\right.$, $\left.H_{\xi_{k}} x\left(i_{k}-\xi_{k-1}\right)\right]^{T} \leq 0$.

Therefore,

$$
\begin{aligned}
\Delta V\left(i_{k}\right) \leq 2 \sum_{\xi_{k}=1}^{d} p_{\xi_{k}}\left[x^{T}\left(i_{k}\right) G^{T}(P+Q) G x\left(i_{k}\right)\right] \\
+2 \sum_{\xi_{k}=1}^{d} p_{\xi_{k}}\left[x^{T}\left(i_{k}-\xi_{i_{k-1}}\right) H_{\xi_{k}}^{T}\right. \\
\left.\times(P+Q) H_{\xi_{k}} x\left(i_{k}-\xi_{i_{k-1}}\right)\right] .
\end{aligned}
$$

Corresponding to (20),

$$
\begin{aligned}
& \Delta V\left(i_{k}\right) \leq \sum_{\xi_{k}=1}^{d} p_{\xi_{k}}\left[x^{T}\left(i_{k}\right)\left[2 G^{T}(P+Q) G-P\right] x\left(i_{k}\right)\right] \\
& \sum_{\xi_{k}=1}^{d}\left[p_{\xi_{k}} x^{T}\left(i_{k}-\xi_{k-1}\right)\left[2 H_{\xi_{k}}^{T}(P+Q) H_{\xi_{k}}-Q\right]\right. \\
&\left.\times x\left(i_{k}-\xi_{k-1}\right)\right] .
\end{aligned}
$$

Denote $\Phi_{i}=2 G^{T}(P+Q) G-P+S+K^{T} R K$.

It is apparently $\Delta V\left(i_{k}\right) \leq 0$, when $(1) \Phi_{i}<0,(2) 2 H_{\xi_{k}}^{T}(P+$ Q) $H_{\xi_{k}}-Q<0$.

Therefore, $E\left(V\left(i_{k}\right)\right) \leq-\min \left\{\lambda_{\min }\left(-\Phi_{i}\right)\right\}\|x(k)\|^{2}$. Denote $\mu=\min \left\{\lambda_{\min }\left(-\Phi_{i}\right)\right\}$

$$
\Xi\left(\sum_{k=0}^{\infty}\left\|x\left(i_{k}\right)\right\|^{2}\right) \leq \frac{1}{\mu} \Xi\left(V\left(x(0), \xi_{0}\right)\right)<\infty .
$$

Then, the networked control systems (10) are stochastic mean-square stable.
Due to (22),

$$
\begin{aligned}
& \sum_{k=0}^{\infty} E\left(V\left(i_{k+1}\right)-V\left(i_{k}\right)\right) \leq-J \\
& \Longrightarrow J \leq V\left(i_{0}\right)-V\left(i_{\infty}\right)<x^{T}\left(i_{0}\right) P x\left(i_{0}\right) \\
&+x^{T}\left(i_{-1}\right) Q x\left(i_{-1}\right) .
\end{aligned}
$$

Theorem 7. For given matrices $R, S$ if there exist matrix $M$ and positive definite matrices $X=P^{-1}, Y=Q^{-1}$ such that

$$
\text { (1) }\left[\begin{array}{cc}
\Xi_{1} & \Xi_{2} \\
* & \Xi_{3}
\end{array}\right]<0 ; \quad \text { (2) }\left[\begin{array}{ccc}
-\frac{1}{2} Y & * & * \\
\Gamma_{1} M+\bar{H} M & -X & 0 \\
\Gamma_{1} M+\bar{H} M & 0 & -Y
\end{array}\right]<0 \text {. }
$$

Then $K=M X^{-1}$ is a guaranteed cost controller gain for the system (10) with disturbance $v_{i_{k}}=0$ and the corresponding closed loop cost function satisfies

$$
J<\lambda_{\max }\left(U^{T} P U\right)+\lambda_{\max }\left(U^{T} Q U\right),
$$

where

$$
\begin{gathered}
\Xi_{1}=\left[\begin{array}{ccc}
-X & * & * \\
A^{\xi_{k}} X+\Gamma_{0} M & -\frac{1}{2} X & 0 \\
A^{\xi_{k}} X+\Gamma_{0} M & 0 & -\frac{1}{2} Y
\end{array}\right], \\
\Xi_{2}=\left[\begin{array}{rr}
M^{T} & 0 \\
0 & 0 \\
0 & 0
\end{array}\right], \quad \Xi_{3}=\left[\begin{array}{cc}
-R^{-1} & 0 \\
* & -S^{-1}
\end{array}\right],
\end{gathered}
$$

$$
\bar{H}=\delta_{\xi_{k}}\left(\sum_{i=0}^{\xi_{k}} A^{i}\right) \Gamma_{2} .
$$

Proof. By Lemma 5, (15) $\Leftrightarrow(28)$,

$$
\text { (1) }\left[\begin{array}{cc}
\Xi_{1}^{\prime} & \Xi_{2}^{\prime} \\
* & \Xi_{3}
\end{array}\right]<0 ; \quad \text { (2) }\left[\begin{array}{ccc}
-\frac{1}{2} Q & H_{\xi_{k_{k}}}^{T} & H_{\xi_{i_{k}}}^{T} \\
* & -P^{-1} & 0 \\
* & * & -Q^{-1}
\end{array}\right]<0 \text {, }
$$

where

$$
\Xi_{1}^{\prime}=\left[\begin{array}{ccc}
-P & G^{T} & G^{T} \\
* & -\frac{1}{2} P^{-1} & 0 \\
* & * & -\frac{1}{2} Q^{-1}
\end{array}\right], \quad \Xi_{2}^{\prime}=\left[\begin{array}{cc}
K^{T} & 0 \\
0 & 0 \\
0 & 0
\end{array}\right] .
$$

Pre- and postmultiplying (28)(1) by $\operatorname{diag}\left(P^{-1}, I, I, I, I\right.$, $I, I)$, Pre- and postmultiplying $(28)(2)$ by $\operatorname{diag}\left(Q^{-1}, I, I\right)$. Define $X=P^{-1}, Y=Q^{-1}$; we obtain (25). 
The initial state of system is unknown; we suppose the initial state of the system (10) is arbitrary and belongs to the set $S=\left\{x\left(-i_{k}\right) \in R^{n}: x\left(-i_{k}\right)=U V\left(i_{k}\right), V^{T}\left(i_{k}\right) V\left(i_{k}\right)<\right.$ $1, k=0,1\}$, where $U$ is a given matrix. Then, the cost bound $J<x^{T}\left(i_{0}\right) P x\left(i_{0}\right)+x^{T}\left(i_{-1}\right) Q x\left(i_{-1}\right)$ leads to $(26)$.

Remark 8. Denote the upper bound of the cost function $J$ as $J^{*}$ and $J^{*}=\theta_{1}+\theta_{2}$ depends on matrices $X, Y$. The optimal guaranteed cost control gain of NCSs (10) can be solved by existing LMI that

$$
\begin{array}{ll}
\text { Minimize } & \theta_{1}+\theta_{2} \\
\text { s.t } & \text { (1) }\left[\begin{array}{cc}
-\theta_{1} I & U^{T} \\
U & -X
\end{array}\right] \leq 0 \\
& \text { (2) }\left[\begin{array}{cc}
-\theta_{2} I & U^{T} \\
U & -Y
\end{array}\right] \leq 0 .
\end{array}
$$

Theorem 9. Take given scalar $\gamma>0$, and matrices $R$, $S$, if there exist matrix $M$ and positive definite matrices $X=P^{-1}, Y=$ $Q^{-1}$ such that

$$
\begin{aligned}
& \text { (1) }\left[\begin{array}{ccc}
\Xi_{1} & \Xi_{2} & \Xi_{4} \\
* & \Xi_{3} & 0 \\
* & * & \Xi_{5}
\end{array}\right]<0, \\
& \text { (2) }\left[\begin{array}{ccc}
-\frac{1}{2} Y & * & * \\
\Gamma_{1} M+\bar{H} M & -X & 0 \\
\Gamma_{1} M+\bar{H} M & 0 & -Y
\end{array}\right]<0,
\end{aligned}
$$

where $\Xi_{4}=\left[\begin{array}{c}X C_{0}^{T} H(C X)^{T} \\ 0\end{array}\right], \Xi_{5}=\operatorname{diag}\left(H^{T} H-\gamma^{2} I,-I\right)$, and $\bar{H}=\delta_{\xi_{k}}\left(\sum_{i=0}^{\xi_{k}} A^{i}\right) \Gamma_{2}$.

Then $K=M X^{-1}$ is a guaranteed cost controller gain for the system (10) with $H_{\infty}$ performance constraint (13) is achieved for all nonzero $v(k)$ and the cost function value is less than a bound:

$$
J<\lambda_{\max }\left(U^{T} P U\right)+\lambda_{\max }\left(U^{T} Q U\right) .
$$

Proof. Consider

$$
\begin{aligned}
\sum_{k=0}^{\infty} E\left(z_{k}^{T} z_{k}\right)-\gamma^{2} v_{k}^{T} v_{k}= & \sum_{k=0}^{\infty} E\left(z_{k}^{T} z_{k}\right)-\gamma^{2} v_{k}^{T} v_{k} \\
& +\Delta V(k)-V_{\infty}+V_{0},
\end{aligned}
$$

$V_{0}=0, V_{\infty}>0$ can be obtained from the zero initial conditions. Therefore,

$$
\begin{aligned}
& \sum_{k=0}^{\infty} E\left(z_{i_{k}}^{T} z_{i_{k}}\right)-\gamma^{2} v_{i_{k}}^{T} v_{i_{k}} \\
& \quad \leq \sum_{k=0}^{\infty} E\left(z_{i_{k}}^{T} z_{i_{k}}\right)-\gamma^{2} v_{i_{k}}^{T} v_{i_{k}}+\Delta V\left(i_{k}\right)
\end{aligned}
$$

$$
\begin{aligned}
& z_{i_{k}}^{T} z_{i_{k}}-\gamma^{2} v_{i_{k}}^{T} v_{i_{k}}+\Delta V\left(i_{k}\right) \\
& \leq \sum_{\xi_{k}=1}^{d} p_{\xi_{k}}\left[x ^ { T } ( i _ { k } ) \left[2 G^{T}(P+Q) G-P+S\right.\right. \\
& \left.\left.+K^{T} R K+C^{T} C\right] x\left(i_{k}\right)\right] \\
& -x^{T}\left(i_{k}\right)\left[S+K^{T} R K\right] x\left(i_{k}\right) \\
& +p_{\xi_{k}} \sum_{\xi_{k}=1}^{d}\left[x^{T}\left(i_{k}\right) C^{T} H v^{T}\left(i_{k}\right)+v^{T}\left(i_{k}\right) H^{T} C x^{T}\left(i_{k}\right)\right. \\
& \left.+v^{T}\left(i_{k}\right) H^{T} H v^{T}\left(i_{k}\right)\right] \\
& +p_{\xi_{k}} \sum_{\xi_{k}=1}^{d}\left[x^{T}\left(i_{k}-\xi_{k-1}\right)\left[3 H_{\xi_{k}}^{T}(P+Q) H_{\xi_{k}}-Q\right]\right. \\
& \left.\quad \times x\left(i_{k}-\xi_{k-1}\right)\right] .
\end{aligned}
$$

By the Schur complement (34) is equivalent to

(1) $\left[\begin{array}{ccc}\Xi_{1}^{\prime} & \Xi_{2}^{\prime} & \Xi_{4} \\ * & \Xi_{3} & 0 \\ * & * & \Xi_{5}\end{array}\right]<0 ; \quad$ (2) $\left[\begin{array}{ccc}-\frac{1}{2} Q & H_{\xi_{i_{k}}}^{T} & H_{\xi_{i_{k}}}^{T} \\ * & -P^{-1} & 0 \\ * & * & -Q^{-1}\end{array}\right]<0$.

Therefore, we can derive $z_{i_{k}}^{T} z_{i_{k}}-\gamma^{2} v_{i_{k}}^{T} v_{i_{k}}+\Delta V\left(i_{k}\right)<0$. Similar to Theorem 6, the networked system (10) is stochastic mean-square stable. Pre- and postmultiplying (35)(1) by $\operatorname{diag}\left(P^{-1}, I, I, I, I, I, I, I\right)$, pre- and postmultiplying (35) (2) by $\operatorname{diag}\left(Q^{-1}, I, I\right)$, and define $X=P^{-1}, Y=Q^{-1}$ we obtain (31).

Similar to Theorem 7, $J<\lambda_{\max }\left(U^{T} P U\right)+\lambda_{\max }\left(U^{T} Q U\right)$.

Remark 10. The optimal guaranteed cost control gain $\left(J^{*}=\right.$ $\theta_{1}+\theta_{2}$ ) in Theorem 9 can be solved by existing LMI that

$$
\text { Minimize } \theta_{1}+\theta_{2}
$$

$$
\begin{array}{ll}
\text { s.t } & \text { (1) }\left[\begin{array}{cc}
-\theta_{1} I & U^{T} \\
U & -X
\end{array}\right] \leq 0 \\
\text { (2) }\left[\begin{array}{cc}
-\theta_{2} I & U^{T} \\
U & -Y
\end{array}\right] \leq 0 .
\end{array}
$$

\section{Numerical Examples}

Example 1. Consider the following system:

$$
\dot{x}=\left[\begin{array}{cc}
-0.3 & -1 \\
0 & -0.1
\end{array}\right] x(t)+\left[\begin{array}{c}
0.01 \\
-0.01
\end{array}\right] u(t) .
$$

The sampling period of controller is $T_{c}=0.01 \mathrm{~s}$, and the sampling period of sensor is $T_{s}=0.15 \mathrm{~s}$. And the packet loss upper bounds $d=2$, and the time delay $\tau=0.03 \mathrm{~s}$. Choose $U=\left[\begin{array}{cc}2.366 & 1.6282 \\ 1.3025 & -1.624\end{array}\right], S=\left[\begin{array}{ll}1 & 0 \\ 0 & 1.1\end{array}\right], R=10$. 
By solving the LMIs given in Theorem 7, we can obtain

$$
X=\left[\begin{array}{ll}
0.1978 & 0.0595 \\
0.0595 & 0.1236
\end{array}\right], \quad M=1.0 e^{-10}\left[\begin{array}{lll}
0.6131 & -0.9766
\end{array}\right] .
$$

Therefore, $K=M X^{-1}=1.0 e^{-008}[0.0640-0.1098]$.

The state responses of closed loop system are shown in Figure 2, where the initial condition is $x(0)=(1,-1)^{T}$. Solving the LMIs given in Remark 8, $J^{*}=51.9179$. The simulation results show that the proposed method is effective.

Example 2. Consider the following system:

$$
\begin{aligned}
\dot{x} & =\left[\begin{array}{cc}
-0.3 & -1 \\
0 & -0.1
\end{array}\right] x(t)+\left[\begin{array}{c}
0.01 \\
-0.01
\end{array}\right] u(t)+\left[\begin{array}{c}
0.1 \\
-0.1
\end{array}\right] v(t), \\
z(t) & =\left[\begin{array}{ll}
1 & 0
\end{array}\right] x(t)+0.091 v(t), \\
v(t) & = \begin{cases}0.1 \sin (t), & 90 \leq t \leq 105, \\
0, & \text { otherwise. }\end{cases}
\end{aligned}
$$

The other parameters are the same with Example 1. Solving the LMIs given in Theorem 9,

$$
\begin{gathered}
X=\left[\begin{array}{ll}
3.9836 & 0.8675 \\
0.8675 & 3.9836
\end{array}\right], \\
M=1.0 e^{-009}[0.5063-0.4462] .
\end{gathered}
$$

And we can obtain $K=M X^{-1}=1.0 e^{-009}$ $[0.1353-0.0375]$. Solving the LMIs given in Remark 10, $J^{*}=$ 63.3589. The state responses of closed loop system are shown in Figure 3, where the initial condition is $x(0)=(1,-1)^{T}$.

Example 3. To illustrate the proposed method's effectiveness, which is obtained in this issue, we consider the following system in $[7,14]$ :

$$
\begin{gathered}
\dot{x}=\left[\begin{array}{cc}
-1.84 & -0.33 \\
7.18 & -1.14
\end{array}\right] x(t)+\left[\begin{array}{c}
2.43 \\
-0.42
\end{array}\right] u(t)+\left[\begin{array}{c}
1.86 \\
-0.76
\end{array}\right] v(t), \\
z(t)=\left[\begin{array}{ll}
0.57 & 0.78
\end{array}\right] x(t)-0.56 v(t) .
\end{gathered}
$$

We can see, $[7,14]$ supposed that the sampling period of controller is $T_{c}=0.01 \mathrm{~s}$, the sampling period of sensor is $T_{s}=0.1 \mathrm{~s}$. And the packet loss upper bounds $d=1$, and the time delay $\tau=0.01 \mathrm{~s}$. Solving the LMIs given in Theorem 9, $K=[0.040,-0.051]$. We can obtain the $H_{\infty}$ bounds from different methods in Table 1. Figure 4 illustrates the merits of the proposed multirate control system both with time delay and packet dropout.

\section{Conclusions}

For multirate NCSs, we mean that the sampling periods of the nodes in the system are not the same. In this paper, the guaranteed cost control for multirate networked control

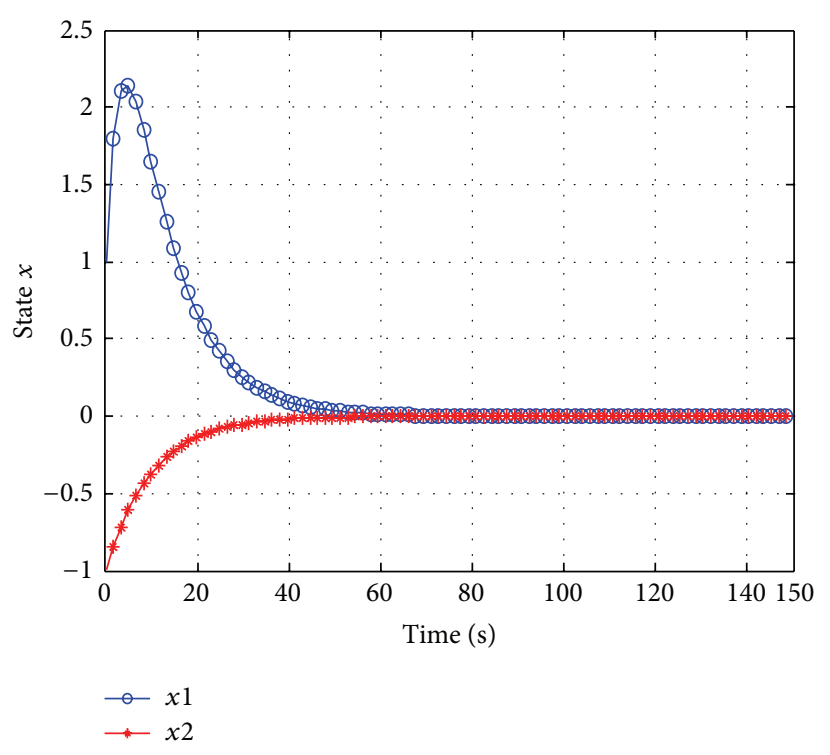

FIGURE 2: The state response of multirate NCSs.

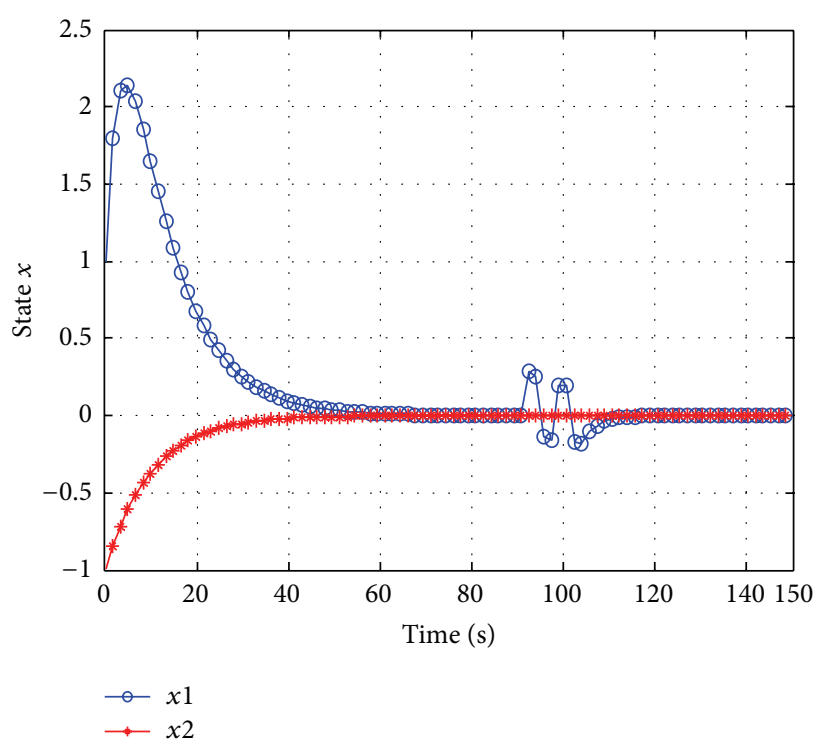

FIGURE 3: The state response of multirate NCSs with noise.

system with both time delay and packet dropout of multirates networked control is discussed. A multirate NCS with simultaneous consideration time delay and packet dropout is modeled as a time-varying sampling system with time delay, in which the newest control inputs are adopted and the Lyapunov function deceasing at each input signal updating point. Numerical examples are given to demonstrate the effectiveness of the proposed method.

The proposed problems in this paper for the nonlinear networked control systems $[40,41]$ have not fully been investigated. The method of fuzzy control $[42,43]$ will be adopted in the future work. 


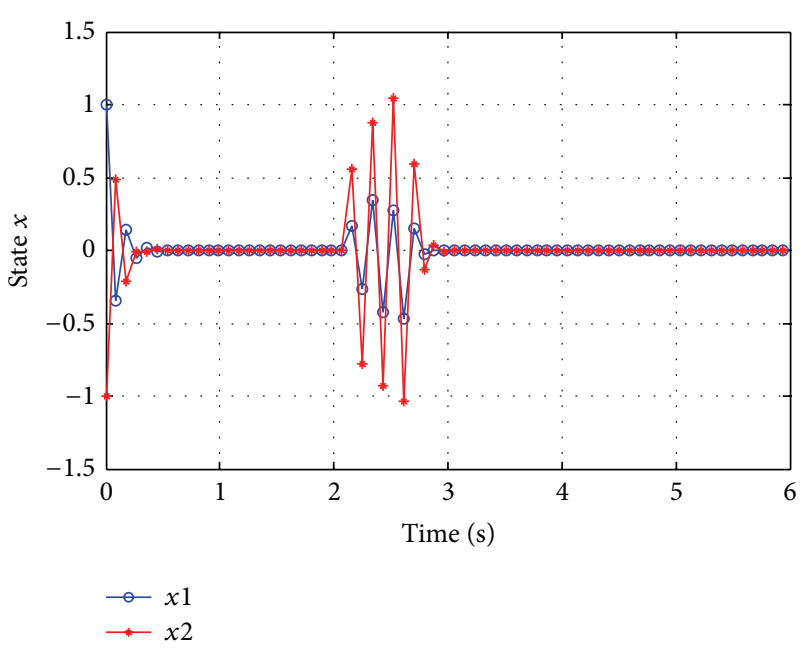

FIGURE 4: The state response of closed loop systems.

TABLE 1: $H_{\infty}$ bounds.

\begin{tabular}{lccc}
\hline Method & Theorem 6 [14] & Corollary 1 [7] & Theorem 9 \\
\hline$\gamma$ & 2.4966 & 4.4967 & 3.8705 \\
\hline
\end{tabular}

\section{Conflict of Interests}

The authors declare that there is no conflict of interests regarding the publication of this paper.

\section{Acknowledgments}

This work was partly supported by National Nature Science Foundation of China $(51375323,61164014)$ and Science and Technology Support Project Plan of Jiangxi Province, China (2010BGB00607).

\section{References}

[1] J. Nilsson, Real-time control systems with delays [Ph.D. thesis], Department of Automatic Control, Lund Institute of Technology, Lund, Sweden, 1998.

[2] Q. X. Zhu and S. S. Hu, "Stochastic optimal control and analysis of stability of networked control systems with long delay," Automatica, vol. 39, no. 11, pp. 1877-1884, 2003.

[3] H. Lin, G. Zhai, and P. J. Antsaklis, "Robust stability and disturbance attenuation analysis of a class of networked control systems," in Proceedings of the 42nd IEEE Conference on Decision and Control, pp. 1182-1187, December 2003.

[4] W. P. M. H. Heemels, A. R. Teel, N. Van De Wouw, and D. Nešić, "Networked control systems with communication constraints: tradeoffs between transmission intervals, delays and performance," IEEE Transactions on Automatic Control, vol. 55, no. 8, pp. 1781-1796, 2010.

[5] A. V. Savkin and T. M. Cheng, "Detectability and output feedback stabilizability of nonlinear networked control systems," IEEE Transactions on Automatic Control, vol. 52, no. 4, pp. 730$735,2007$.

[6] L. Q. Zhang, Y. Shi, T. W. Chen, and B. Huang, "A new method for stabilization of networked control systems with random delays," IEEE Transactions on Automatic Control, vol. 50, no. 8, pp. 1177-1181, 2005.

[7] Y.-L. Wang and G.-H. Yang, "Multiple communication channels-based packet dropout compensation for networked control systems," IET Control Theory and Applications, vol. 2, no. 8, pp. 717-727, 2008.

[8] M. Yu, L. Wang, G. M. Xie, and T. G. Chu, "Stabilization of networked control systems with data packet dropout via switched system approach," in Proceedings of the IEEE International Symposium on Computer Aided Control System Design, pp. 362-367, September 2004.

[9] W. Zhang, M. S. Branicky, and S. M. Phillips, "Stability of networked control systems," IEEE Control Systems Magazine, vol. 21, no. 1, pp. 84-97, 2001.

[10] A. S. Matveev and A. V. Savkin, "Almost sure nonobservability and unstabilizability of unstable noisy linear plants via communication channels with packet losses," in Proceedings of the 44th IEEE Conference on Decision and Control, and the European Control Conference (CDC-ECC '05), pp. 7338-7341, December 2005.

[11] Y. Liang, T. Chen, and Q. Pan, "Optimal linear state estimator with multiple packet dropouts," IEEE Transactions on Automatic Control, vol. 55, no. 6, pp. 1428-1433, 2010.

[12] T. Liu, H. Zhang, and Q. J. Chen, "Control for networked control systems with communication constraints," Control and Decision, vol. 28, no. 4, pp. 537-546, 2013.

[13] P. Shi, Y. Q. Xia, G. P. Liu, and D. Rees, "On designing of slidingmode control for stochastic jump systems," IEEE Transactions on Automatic Control, vol. 51, no. 1, pp. 97-103, 2006.

[14] Y.-L. Wang and G.-H. Yang, " $\mathrm{H}_{\infty}$ control of networked control systems with delay and packet disordering via predictive method," in Proceedings of the American Control Conference (ACC '07), pp. 1021-1026, July 2007.

[15] W.-A. Zhang and L. Yu, "Modelling and control of networked control systems with both network-induced delay and packetdropout," Automatica, vol. 44, no. 12, pp. 3206-3210, 2008.

[16] J. Wu and T. W. Chen, "Design of networked control systems with packet dropouts," IEEE Transactions on Automatic Control, vol. 52, no. 7, pp. 1314-1319, 2007.

[17] M. Yu, L. Wang, T. G. Chu, and F. Hao, "Stabilization of networked control systems with data packet dropout and transmission delays: continuous-time case," European Journal of Control, vol. 11, no. 1, pp. 40-49, 2005.

[18] X. He, Z. Wang, and D. H. Zhou, "Robust fault detection for networked systems with communication delay and data missing," Automatica, vol. 45, no. 11, pp. 2634-2639, 2009.

[19] S. S. L. Chang and T. K. C. Peng, "Adaptive guaranteed cost control of systems with uncertain parameters," IEEE Transactions on Automatic Control, vol. 17, no. 4, pp. 474-483, 1972.

[20] G.-R. Matías and B. Antonio, "Analysis of networked control systems with drops and variable delays," Automatica, vol. 43, no. 12, pp. 2054-2059, 2007.

[21] I. R. Petersen and D. C. McFarlane, "Optimal guaranteed cost control and filtering for uncertain linear systems," IEEE Transactions on Automatic Control, vol. 39, no. 9, pp. 1971-1977, 1994.

[22] L. Yu and J. Chu, "LMI approach to guaranteed cost control of linear uncertain time-delay systems," Automatica, vol. 35, no. 6, pp. 1155-1159, 1999.

[23] B. Chen and X. Liu, "Fuzzy guaranteed cost control for nonlinear systems with time-varying delay," IEEE Transactions on Fuzzy Systems, vol. 13, no. 2, pp. 238-249, 2005. 
[24] H. G. Zhang, D. D. Yang, and T. Y. Chai, "Guaranteed cost networked control for T-S fuzzy systems with time delays," IEEE Transactions on Systems, Man and Cybernetics Part C, vol. 37, no. 2, pp. 160-172, 2007.

[25] J. H. Park and H. Y. Jung, "On the design of nonfragile guaranteed cost controller for a class of uncertain dynamic systems with state delays," Applied Mathematics and Computation, vol. 150, no. 1, pp. 245-257, 2004.

[26] H. Lin and P. J. Antsaklis, "Stability and persistent disturbance attenuation properties for a class of networked control systems: switched system approach," International Journal of Control, vol. 78, no. 18, pp. 1447-1458, 2005.

[27] Q. X. Zhu, H. L. Liu, and S. S. Hu, "Uniformed model of networked control systems with long time delay," Journal of Systems Engineering and Electronics, vol. 19, no. 2, pp. 385-390, 2008.

[28] L. Y. Xiang and G. Guo, "Model-based networked control system with multi-rate sampling," Information and Control, vol. 34, no. 3, pp. 373-378, 2005 (Chinese).

[29] D. M. Tilbury and D. Georgiev, "Packet-based control: the ${ }_{\mathrm{H}^{-}}{ }^{-}$ optimal solution," Automatica, vol. 42, no. 1, pp. 137-144, 2006.

[30] V. H. Nguyen and Y. S. Suh, "A modified multirate controller for networked systems with a send-on-delta transmission method," in Proceedings of the $3 \mathrm{rd}$ International Conference on Intelligent Computing, pp. 304-315, 2007.

[31] J. Jin, W. Zhiwen, and L. Dongsong, "Model-based control scheme for networked multi-rate sampling systems," in Proceedings of the International Conference on Measuring Technology and Mechatronics Automation (ICMTMA '09), pp. 812-815, April 2009.

[32] Z. H. Guan, C. -X. Yang, and J. Huang, "Stabilization of networked control systems with random delays: a new multirate method," in Proceedings of the 17th World Congress of IFAC, pp. 4204-4209, Seoul, Korea, July 2008.

[33] Y. Liang, T. Chen, and Q. Pan, "Optimal linear state estimator with multiple packet dropouts," IEEE Transactions on Automatic Control, vol. 55, no. 6, pp. 1428-1433, 2010.

[34] D. X. Wu, J. Wu, S. Chen, and J. Chu, "Optimal linear state estimator with multiple packet dropouts," IEEE Transactions on Automatic Control, vol. 55, no. 5, pp. 1202-1208, 2010.

[35] Q. X. Zhu, "Controllability of multi-rate networked control systems with short time delay," Advanced Materials Research, vol. 219-220, pp. 855-859, 2011.

[36] Q. X. Zhu, "Observability of multi-rate networked control systems with short time delay," Communications in Computer and Information Science, vol. 144, no. 2, pp. 396-401, 2011.

[37] S. Yin, S. Ding, and H. Luo, "Real-time implementation of fault tolerant control system with performance optimization," IEEE Transactions on Industrial Electronics, vol. 61, no. 5, pp. 24022411, 2013.

[38] S. Yin, S. Ding, A. Haghani, and H. Hao, "Data-driven monitoring for stochastic systems and its application on batch process," International Journal of Systems Science, vol. 44, no. 7, pp. 13661376, 2013.

[39] S. Yin, S. Ding, A. Haghani, H. Hao, and P. Zhang, "A comparison study of basic data driven fault diagnosis and process monitoring methods on the benchmark Tennessee Eastman process," Journal of Process Control, vol. 22, no. 9, pp. 1567-1581, 2012.

[40] H. J. Gao, T. W. Chen, and J. Lam, "A new delay system approach to network-based control," Automatica, vol. 44, no. 1, pp. 39-52, 2008.
[41] H. J. Gao and T. W. Chen, "New results on stability of discretetime systems with time-varying state delay," IEEE Transactions on Automatic Control, vol. 52, no. 2, pp. 328-334, 2007.

[42] H. J. Li, H. H. Liu, H. J. Gao, and P. Shi, "Reliable fuzzy control for active suspension systems with actuator delay and fault," IEEE Transactions on Fuzzy Systems, vol. 20, no. 2, pp. 342-357, 2012.

[43] H. Y. Li, J. Y. Yu, C. Hilton, and H. H. Liu, "Adaptive slidingmode control for nonlinear active suspension vehicle systems using T-S fuzzy approach," IEEE Transactions on Industrial Electronics, vol. 60, no. 8, pp. 3328-3338, 2013. 


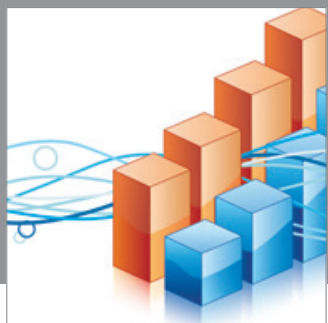

Advances in

Operations Research

mansans

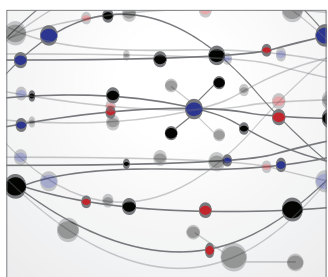

The Scientific World Journal
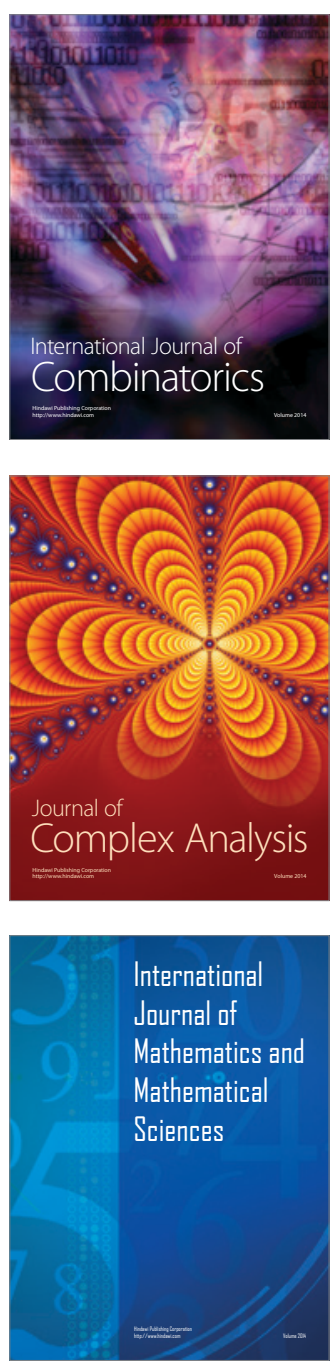
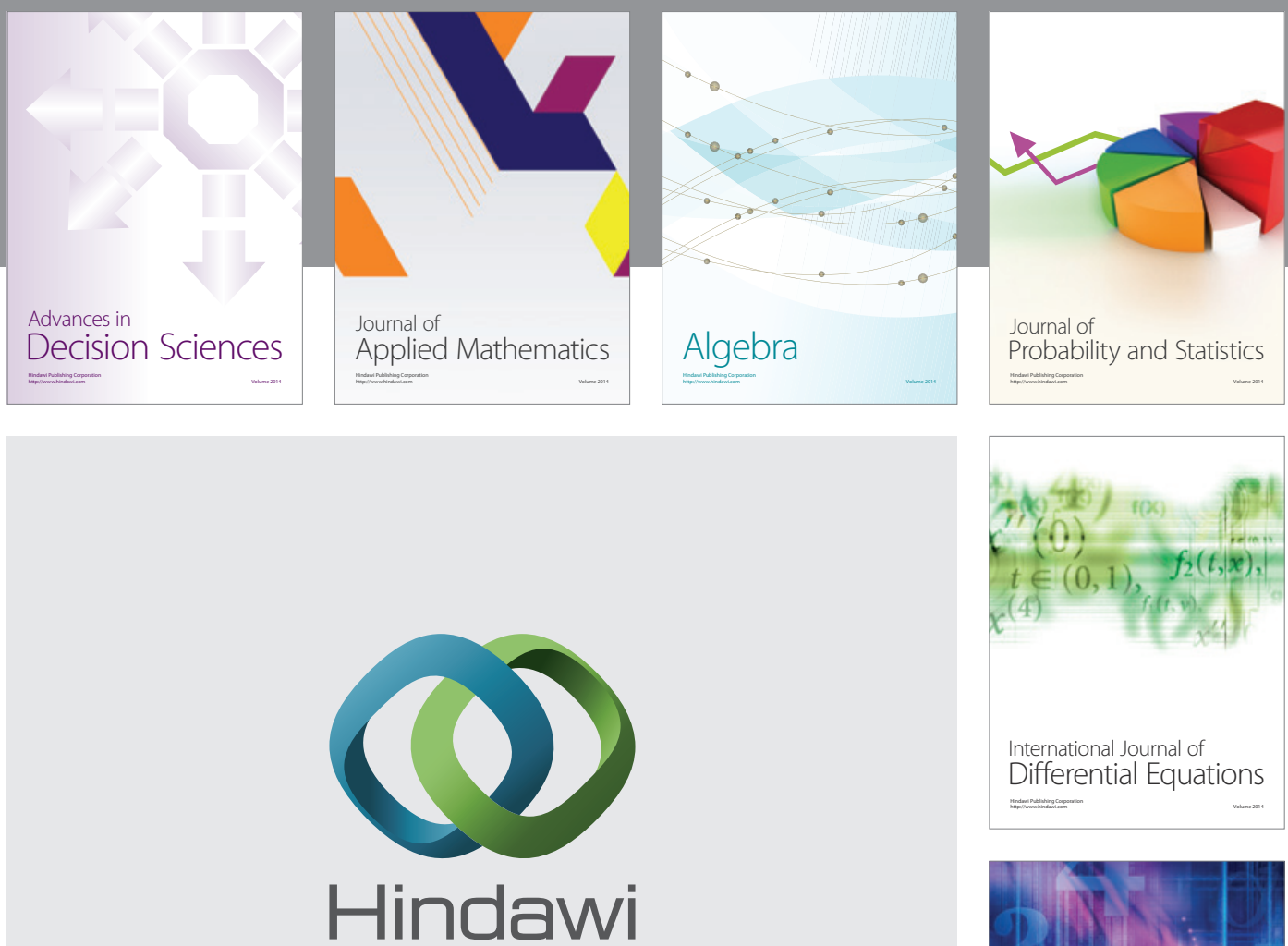

Submit your manuscripts at http://www.hindawi.com
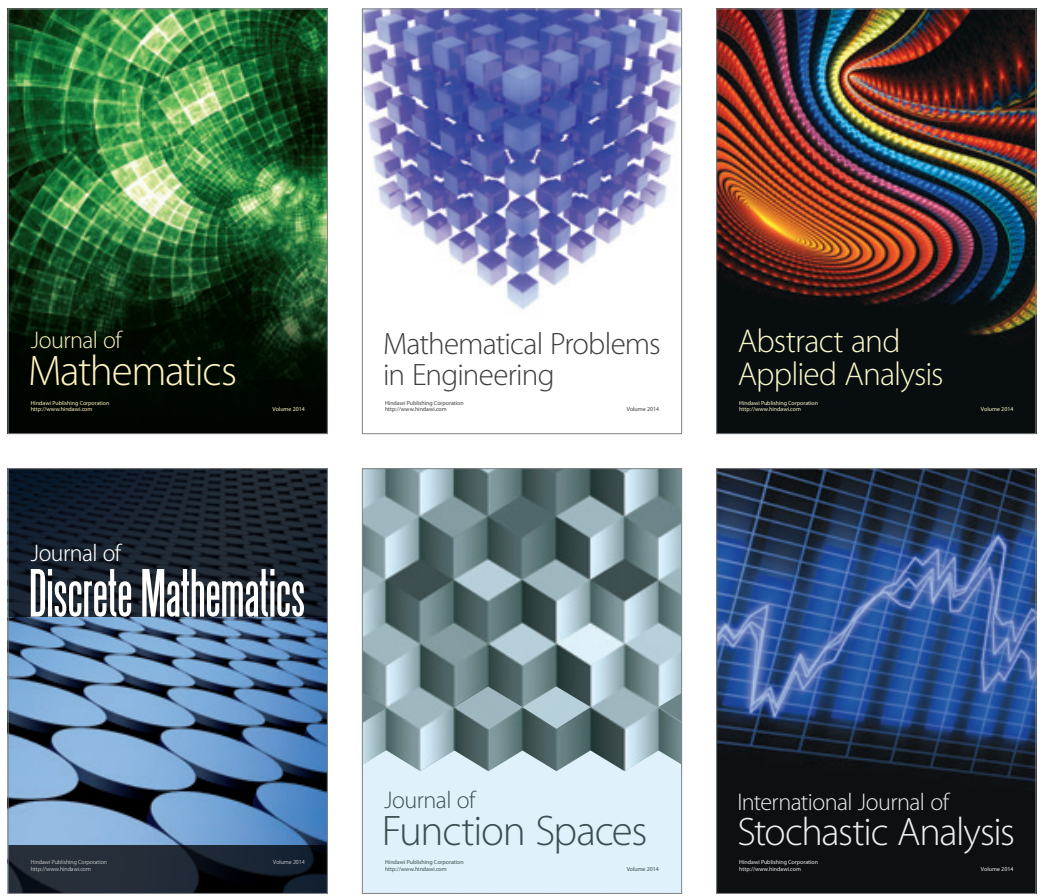

Journal of

Function Spaces

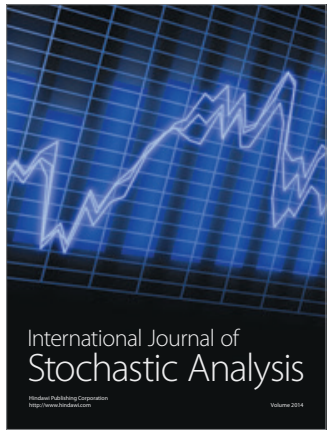

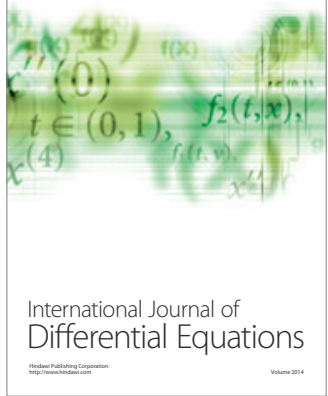
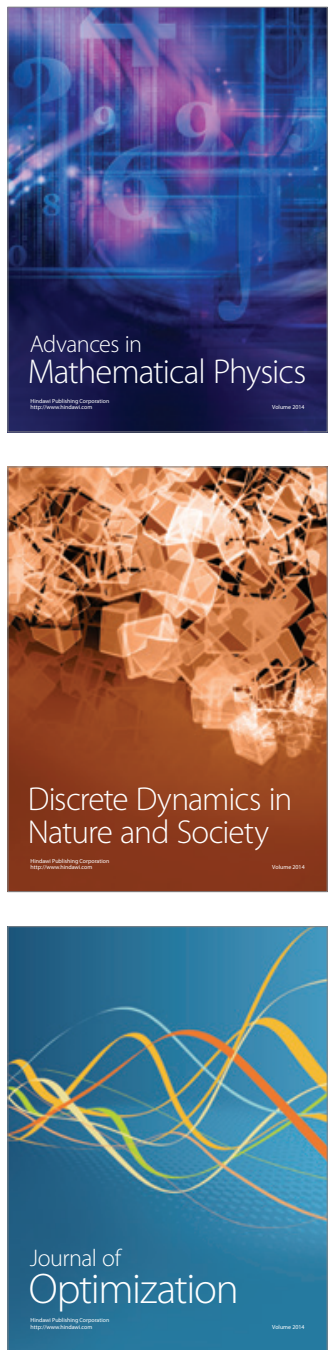